\title{
Benzocaína e eugenol como anestésico para juvenis de pacu (Piaractus
}

\section{mesopotamicus)}

\author{
Benzocaine and eugenol as anesthetic for juvenile pacu (Piaractus mesopotamicus) \\ Benzocaína y eugenol como anestésico para pacu juvenil (Piaractus mesopotamicus)
}

Recebido: 25/06/2021 | Revisado: 04/07/2021 | Aceito: 06/07/2021 | Publicado: 16/07/2021

Bruno Estevão de Souza

ORCID: https://orcid.org/0000-0002-1831-0061 Instituto Federal de Educação Ciência e Tecnologia do Paraná, Brasil

E-mail: bruno.souza@ifpr.edu.br

Odair Diemer

ORCID: https://orcid.org/0000-0003-3527-2455 Instituto Federal de Educação Ciência e Tecnologia de Mato Grosso do Sul, Brasil E-mail: odair.diemer@ifms.edu.br

Arcangelo Augusto Signor

ORCID: https://orcid.org/0000-0003-4686-3488 Instituto Federal de Educação Ciência e Tecnologia do Paraná, Brasil E-mail: arcangelo.signor@ifpr.edu.br

Wilson Rogerio Boscolo

ORCID: https://orcid.org/0000-0002-1808-0518

Universidade Estadual do Oeste do Paraná, Brasil E-mail: wilsonboscolo@hotmail.com

Aldi Feiden

ORCID: https://orcid.org/0000-0002-6823-9291

Universidade Estadual do Oeste do Paraná, Brasil E-mail: aldifeiden@gmail.com

\begin{abstract}
Resumo
O presente trabalho teve como objetivo avaliar a influência das concentrações da benzocaína e eugenol como anestésico na indução e recuperação de juvenis de pacu (P. mesopotamicus). Foram utilizados 288 juvenis de pacu em um delineamento inteiramente casualizado constituído de 12 concentrações de benzocaína $(12,5 ; 25 ; 37,5 ; 50 ; 62,5$; $75 ; 87,5 ; 100 ; 112,5 ; 125 ; 137,5$ e 150 mg.. $\left.\mathrm{L}^{-1}\right)$ e 11 concentrações de eugenol $(7,5 ; 15 ; 22,5 ; 30 ; 37,5 ; 45 ; 52,5 ; 60$; 67,$5 ; 75$ e $82,5 \mathrm{mg} . \mathrm{L}^{-1}$ ) e um grupo controle. Os peixes foram divididos em 24 lotes, com 12 peixes escolhidos aleatoriamente e expostos individualmente para cada concentração de anestésico em um aquário de vidro com volume útil de 30L, mas contendo apenas 10L de água. A benzocaína mostrou-se eficiente para a anestesia em juvenis de pacu nas distintas concentrações, exceto para os valores 12,5 e $25 \mathrm{mg} . \mathrm{L}^{-1}$. O eugenol mostrou-se eficaz para a anestesia, exceto para os valores 7,5 e $15 \mathrm{mg} \cdot \mathrm{L}^{-1}$. A melhor dose de benzocaína e eugenol para sedação e recuperação é de 75 mg. $\mathrm{L}^{-1}$ e $52,5 \mathrm{mg} . \mathrm{L}^{-1}$, respectivamente.
\end{abstract}

Palavras-chave: Aquicultura; Cultivo intensivo; Manejo de peixe.

\begin{abstract}
This study aimed to evaluate the influence of benzocaine and eugenol concentrations as anesthetics on the induction and recovery of juvenile pacu ( $P$. mesopotamicus). 288 juvenile pacu were used in a completely randomized design consisting of 12 concentrations of benzocaine $(12.5 ; 25 ; 37.5 ; 50 ; 62.5 ; 75 ; 87.5 ; 100 ; 112.5 ; 125 ; 137,5$ and 150 mg.L $\left.\mathrm{L}^{-1}\right)$ and 11 concentrations of eugenol $(7.5 ; 15 ; 22.5 ; 30 ; 37.5 ; 45 ; 52.5 ; 60 ; 67.5 ; 75$ and $82.5 \mathrm{mg}$. L-1) and a control group. The fish were divided into 24 lots, with 12 fish chosen at random and individually exposed to each anesthetic concentration in a glass aquarium with a useful volume of $30 \mathrm{~L}$, but containing only $10 \mathrm{~L}$ of water. Benzocaine proved to be efficient for anesthesia in juvenile pacu at different concentrations, except for values 12.5 and $25 \mathrm{mg} . \mathrm{L}^{-1}$. Eugenol was effective for anesthesia, except for the values 7.5 and $15 \mathrm{mg} . \mathrm{L}^{-1}$. The best dose of benzocaine and eugenol for sedation and recovery is $75 \mathrm{mg} . \mathrm{L}^{-1}$ and $52.5 \mathrm{mg} . \mathrm{L}^{-1}$, respectively.
\end{abstract}

Keywords: Aquaculture; Intensive farming; Fish management.

\section{Resumen}

Este estudio tuvo como objetivo evaluar la influencia de las concentraciones de benzocaína y eugenol como anestésicos en la inducción y recuperación del pacu juvenil ( $P$. mesopotamicus). Se utilizaron 288 pacu juveniles en un diseño completamente aleatorizado que consta de 12 concentraciones de benzocaína $(12,5 ; 25 ; 37,5 ; 50 ; 62,5 ; 75$; 87,$5 ; 100 ; 112,5 ; 125 ; 137,5$ y 150 mg.L $\left.\mathrm{L}^{-1}\right)$ y 11 concentraciones de eugenol $(7.5 ; 15 ; 22.5 ; 30 ; 37.5 ; 45 ; 52.5 ; 60$; 
67.5; 75 y $82.5 \mathrm{mg} . \mathrm{L}^{-1}$ ) y un grupo control. Los peces se dividieron en 24 lotes, con 12 peces elegidos al azar y expuestos individualmente a cada concentración de anestésico en un acuario de vidrio con un volumen útil de 30 litros, pero que contenía solo 10 litros de agua. La benzocaína demostró ser eficaz para la anestesia en pacu juvenil a diferentes concentraciones, excepto en los valores de 12,5 y $25 \mathrm{mg} . \mathrm{L}^{-1}$. El eugenol demostró ser efectivo para la anestesia, excepto para los valores 7.5 y $15 \mathrm{mg} . \mathrm{L}^{-1}$. La mejor dosis de benzocaína y eugenol para la sedación y la recuperación es $75 \mathrm{mg} . \mathrm{L}^{-1}$ y $52,5 \mathrm{mg} . \mathrm{L}^{-1}$, respectivamente.

Palabras clave: Acuicultura; Cultivo intensivo; Manejo de peces.

\section{Introdução}

O pacu (Piaractus mesopotamicus) é um peixe muito encontrado nas bacias dos rios Paraná, Uruguai e Paraguai (Nakatani et al., 2001) apresenta potencial para a piscicultura intensiva devido à fácil adaptação (Fernandes et al., 2000), rusticidade, boas taxas de crescimento e aceitação pelo mercado consumidor (Jomori et al., 2005), e rendimento de carcaça (Faria et al., 2003, Bombardelli et al., 2007) em tronco limpo que é a parte do pescado para a indústria (Signor et al., 2010).

No entanto, de maneira contrária as suas excelentes características para aqüicultura continental, o pacu durante as práticas rotineiras de manejo, apresenta-se agitado, tornando sua manipulação arriscada devido ao fato de expor os peixes a uma variedade de fatores estressantes, principalmente na fase jovem. Os organismos expostos a estes estímulos estão propensos a alterações fisiológicas e comportamentais, que refletem na redução do consumo de alimento e retardamento do crescimento, o que predispõe a ocorrência de infecções e a mortalidade dos animais (Anziliero et al., 2008). No cultivo de peixes, as principais situações estressantes são impostas aos animais, como os procedimentos de biometria, transporte e reprodução artificial. Os efeitos destes manejos vão desde perda do peso, apetite, baixo crescimento, acometimento de doenças até a morte dos indivíduos (Vidal et al., 2008). Desta forma, o uso de anestésicos é imprescindível para reduzir o estresse e a mortalidade durante as rotinas de manejo, e segurança durante práticas envolvendo manipulação.

A aquicultura tem passado por inúmeras alterações nos últimos anos, sendo a intensificação dos sistemas produtivos em viveiros escavados ou o uso de tanques-redes, assim como possibilidade de uso de bioflocos como sistema produtivo com o foco na produtividade com redução dos impactos ambientais. A piscicultura intensiva em viveiros escavados, tem proporcionado aumento dos resíduos como nitrogênio e fosforo, em função dos usos de rações proporcionados pela intensificação (Coldebella et al., 2020) tornando a busca pelo equilíbrio entre produção e geração de resíduos algo a ser implementado. A criação de peixes em tanques-rede exige maior conhecimento técnico do produtor, rações mais bem elaboradas, manejos tecnológicos específicos, ou seja, maiores custos, no entanto a produtividade é bem superior, e consequentemente se bem conduzida pelo produtor trará maior lucratividade (Signor et al., 2020). A tecnologia da produção em bioflocos, pode resultar em maior produtividade com menor impacto sobre o meio ambiente, segundo Tavares et al. (2021), a sustentabilidade econômica, social e ambiental em sistemas de produção de peixes através da tecnologia bioflocos, especificamente para a espécie Oreochromis niloticus, envolve o desenvolvimento técnico e manejo adequado.

Contudo, o bem-estar animal deve ser considerado em qualquer modelo produtivo, onde o uso de anestésicos auxiliam na redução do estresse provocado pelos manejos intensos da piscicultura. A escolha de um anestésico deve ser fundamentada em critérios como eficácia, custo, disponibilidade no mercado, segurança durante o uso e possíveis efeitos colaterais aos peixes, aos seres humanos e ao meio ambiente. Dentre os diversos anestésicos utilizados no Brasil, destacam-se a benzocaína e o eugenol.

A benzocaína se destaca, principalmente, pela fácil aquisição, menor custo e segurança ao usuário, atendendo a maior parte dos critérios estabelecidos por Ross e Ross (1999), para um anestésico ideal para peixes. Ela não provoca diminuição no crescimento, nem problemas na reprodução em espécies testadas até o momento. É um anestésico de uso humano e as doses utilizadas em peixes não causam nenhum dano ao operador (Roubach \& Gomes, 2001). 
O eugenol, principal componente do óleo de cravo é um produto natural extraídos de plantas amazônicas. Entre as características desejáveis, o eugenol apresenta rapidez de ação, já que induz rapidamente à anestesia profunda, diminuindo os efeitos do estresse causado pelo manejo (Hajek et al., 2006). Em comparação com outros anestésicos, parece ser mais prático a campo, porque tem a capacidade de imobilizar os peixes rapidamente, quando utilizado em baixas concentrações. Outra vantagem é a sua rápida metabolização e excreção pelos peixes, não exigindo tempo de carência quando estes são destinados ao consumo humano (Hisano et al., 2008). Contudo, ainda não existe regulamentação para o uso desse medicamento em peixes.

O procedimento de anestesia, por ser uma operação muitas vezes necessária na aquicultura, deve ser desempenhado de modo mais eficiente possível, tanto do ponto de vista biológico, quanto econômico. O emprego de uma concentração certa de anestésico é fundamental para evitar desperdício do produto ou a morte dos peixes por superdose. Do ponto de vista biológico o procedimento tem por finalidade anestesiar os peixes sem originar nenhum problema no crescimento e na reprodução (Roubach \& Gomes, 2001).

Cada anestésico exige concentração diferente para induzir ao estágio anestésico desejado, sendo indispensável testar várias doses antes do tratamento definitivo, para não causar mortalidade dos animais submetidos ao tratamento. Neste sentido, o presente trabalho teve por objetivo avaliar a influência das concentrações de benzocaína e eugenol como anestésico na indução e recuperação de juvenis de pacu (P. mesopotamicus).

\section{Metodologia}

O trabalho foi realizado no Laboratório de Aquicultura da Universidade Estadual do Oeste do Paraná - UNIOESTE, Campus de Toledo, Paraná, Brasil.

Foram utilizados 288 juvenis de pacu (Piaractus mesopotamicus) com peso de 6,28 $\pm 2,22 \mathrm{~g}$ e comprimento total 6,94 $\pm 0,93 \mathrm{~cm}$ adquiridos em uma piscicultura comercial localizada na região Oeste do estado do Paraná. Na piscicultura os juvenis foram acondicionados em sacos plásticos (10L de água os quais foram inflados com $20 \mathrm{~L}$ de oxigênio puro), amarrados com tiras de borracha e acondicionados dentro de caixas de papelão. Após estes procedimentos os animais foram submetidos a uma viagem de carro de aproximadamente 1 hora.

Após a chegada, os peixes foram aclimatados durante sete dias em uma caixa d'água circular com volume de 2000L com aeração e renovação de água constante e um sistema de aquecimento com resistência e termostato mantendo a temperatura da água em $25^{\circ} \mathrm{C}$. Os animais foram alimentados até a saciedade aparente duas vezes ao dia com ração comercial extrusada com $40 \%$ de proteína bruta.

Após o período de aclimatação o experimento de anestesia foi conduzido em aquário de vidro com volume útil de 30L, mas contendo apenas $10 \mathrm{~L}$ de água com as respectivas doses de anestésico e aeração constante.

O delineamento experimental foi constituído de 24 tratamentos, correspondentes a 12 concentrações do anestésico benzocaína $\left(12,5 ; 25 ; 37,5 ; 50 ; 62,5 ; 75 ; 87,5 ; 100 ; 112,5 ; 125 ; 137,5\right.$ e $\left.150 \mathrm{mg} . \mathrm{L}^{-1}\right)$, e a 11 concentrações do anestésico eugenol $\left(7,5 ; 15 ; 22,5 ; 30 ; 37,5 ; 45 ; 52,5 ; 60 ; 67,5 ; 75\right.$ e $\left.82,5 \mathrm{mg} . \mathrm{L}^{-1}\right)$ e um grupo controle. Para os anestésicos e as concentrações foram utilizados 12 peixes escolhidos aleatoriamente $(n=12)$ e expostos individualmente para cada concentração. A água dos aquários foi renovada ao término de cada concentração testada.

O grupo controle recebeu o mesmo manejo, porém em água sem anestésico para monitoramento dos parâmetros comportamentais e possível mortalidade durante e após os procedimentos. Os tempos necessários para o aparecimento dos padrões comportamentais avaliados foram monitorados por meio de cronômetro digital (Tabela 1), através de observações qualitativas. Porém, através destas observações foram tomadas características de comportamentos e transformadas em medidas quantitativa (Pereira et al., 2018), para posteriores análises estatísticas. 
Tabela 1. Características comportamentais dos peixes de acordo com os diferentes estágios de anestesia.

\begin{tabular}{cc}
\hline Estágio & Característica de comportamento \\
\hline I & Movimento opercular visivelmente lento ou errático \\
II & Perda parcial de equilíbrio e dificuldade de manter posição normal de nado, quando parado \\
III & Perda total de equilíbrio e incapacidade de recuperar a posição vertical de nado (“barriga para \\
IV & cima”) \\
Recuperado & Recuperação da posição normal de nado e da capacidade de nadar
\end{tabular}

Fonte: Woody et al. (2002).

A ausência de reação a qualquer estímulo foi verificada pelo toque na lateral dos peixes com um bastão de vidro. Quando os animais atingiam o estágio IV (Tabela 1). Foram retirados do aquário de indução, secos com papel toalha, medidos e pesados a fim de simular o processo de manejo, depois transferidos para o aquário de recuperação.

A recuperação anestésica dos peixes foi realizada individualmente, em um aquário de vidro com volume útil de 30L, mas contendo apenas 20L de água, com aeração constante e sem anestésico, onde os peixes eram acondicionados e o tempo de recuperação aferido.

Após o restabelecimento dos indivíduos no aquário de recuperação, eles foram transferidos para aquários de 30L onde receberam alimentação e ficaram em observação durante 96 horas após indução anestésica para o monitoramento da mortalidade (Vidal et al., 2006).

Para a utilização dos anestésicos (benzocaína e o eugenol) foram diluídos em álcool etílico $\left(92,8^{\circ}\right)$, o que resultou em solução estoque na proporção de $100 \mathrm{mg} \cdot \mathrm{mL}^{-1}(1: 10)$.

Para a análise estatística do efeito da concentração da benzocaína e do eugenol sobre os peixes foi comparada a média dos valores encontrados por meio de análise de variância (ANOVA) com nível de significância de 5\% e no caso de diferenças estatísticas foi aplicado o teste de Tukey em $5 \%$ de probabilidade.

\section{Resultados e Discussão}

Durante o experimento os valores da temperatura, oxigênio dissolvido, $\mathrm{pH}$ e condutividade elétrica da água foram de 25,42 ${ }^{\circ} \mathrm{C}, 5,32 \mathrm{mg} . \mathrm{L}^{-1}, 7,42$ e $41 \mu \mathrm{S} . \mathrm{cm}^{-1}$, respectivamente, permaneceram dentro de faixas recomendadas para a espécie (Urbinati \& Gonçalves, 2005).

A benzocaina apresentou efeito positivo na indução e na recuperação, pois com o aumento da concentração apresentou redução no tempo de indução e de recuperação (Figura 1). 
Figura 1. Indução e recuperação da benzocaina para juvenis de pacu.

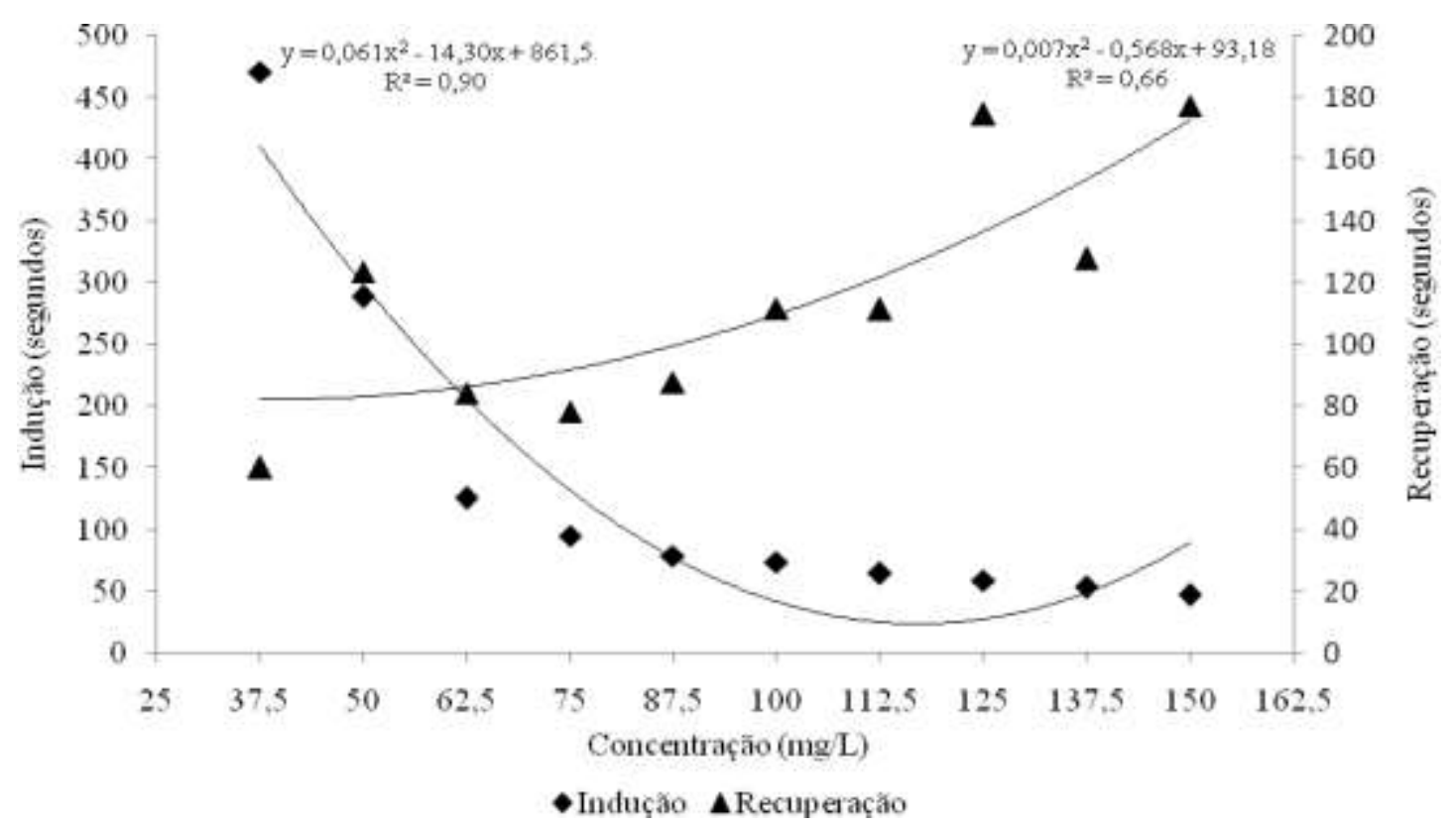

Indução: $y=0,007 x^{2}-0,568 x+93,18, R^{2}=0,659$. Recuperação: $y=0,061 x^{2}-14,30 x+861,5, R^{2}=0,900$. Fonte: Autores.

A benzocaína mostrou-se eficiente para a anestesia em juvenis de pacu nas distintas concentrações analisadas, entretanto, os valores inferiores a 12,5 e $25 \mathrm{mg} . \mathrm{L}^{-1}$, não foram suficiente para a anestesia completa dos animais. Contudo a melhor dosagem dessa substância para indução e recuperação é de 75 mg.L -1 $^{-1}$ (Tabela 2). Após as 96 horas de acompanhamento não foi verificado mortalidade dos peixes submetidos à anestesia com benzocaína.

Tabela 2. Tempo de indução e recuperação de juvenis de pacu expostos as diferentes concentrações da benzocaína.

\begin{tabular}{lcc}
\hline Concentração (mg.L $\left.{ }^{-1}\right)$ & Indução (segundos) & Recuperação (segundos) \\
\hline 37,5 & $471,17 \pm 81,41 \mathrm{a}$ & $60,16 \pm 16,26 \mathrm{~b}$ \\
50,0 & $288,33 \pm 67,37 \mathrm{~b}$ & $123,58 \pm 40,82 \mathrm{ab}$ \\
62,5 & $125,83 \pm 31,63 \mathrm{c}$ & $84,40 \pm 27,11 \mathrm{ab}$ \\
75,0 & $95,08 \pm 7,27 \mathrm{~cd}$ & $78,25 \pm 8,25 \mathrm{~b}$ \\
87,5 & $78,91 \pm 8,56 \mathrm{~cd}$ & $87,75 \pm 14,64 \mathrm{ab}$ \\
100,0 & $74,08 \pm 8,21 \mathrm{~d}$ & $111,75 \pm 66,10 \mathrm{ab}$ \\
112,5 & $64,25 \pm 6,03 \mathrm{~d}$ & $111,58 \pm 34,10 \mathrm{ab}$ \\
125,0 & $58,91 \pm 6,24 \mathrm{~d}$ & $174,75 \pm 133,24 \mathrm{ab}$ \\
137,5 & $53,33 \pm 8,81 \mathrm{~d}$ & $128 \pm 57,31 \mathrm{ab}$ \\
150,0 & $47,25 \pm 7,96 \mathrm{~d}$ & $177,5 \pm 155,30 \mathrm{a}$ \\
\hline C.V. (\%) & 26,12 & 64,37 \\
\hline
\end{tabular}

Medias na mesma coluna seguidos de mesma letra não diferem estatisticamente $(\mathrm{P}<0,05)$ pelo teste de Tukey. Fonte: Autores.

As respostas do pacu corroboram com os estudos de Okamoto et al. (2009) que avaliaram a eficácia da benzocaína e do eugenol como anestésicos para juvenis de pampo (Trachinotus marginatus). Os autores relatam que a dose de 50 mg.L ${ }^{-1}$ de 
benzocaína proporcionou um período de latência e recuperação dentro da faixa considerada adequada, concentração observada de $75 \mathrm{mg} . \mathrm{L}^{-1}$ para juvenis de pacu.

Avaliando diferentes concentrações de benzocaína na indução anestésica do lambari-do-rabo amarelo (Astyanax altiparanae), a taxa de sobrevivência pós-indução foi muito diferente entre as concentrações (Gimbo et al., 2008). Estes observaram que a concentração mais baixa $\left(50 \mathrm{mg} \cdot \mathrm{L}^{-1}\right)$ resultou em maior tempo necessário à anestesia mas por outro lado, para a concentração mais alta (125 mg. $\left.\mathrm{L}^{-1}\right)$, o tempo necessário à indução foi menor, ocasionando, contudo, uma elevada mortalidade sete dias após a indução anestésica (72,5\%). Discordando dos resultados apresentados neste trabalho onde não foi verificada mortalidade dos peixes submetidos a diferentes concentrações de benzocaína. Todavia, corroborando com Okamoto et al, (2009) que não verificaram mortalidade de juvenis de pampo (Trachinotus marginatus) durante e após os testes.

O período necessário para se alcançar à recuperação foi estatisticamente $(\mathrm{P}<0,05)$ influenciado pela concentração da benzocaína. Entretanto, os peixes expostos à dose de benzocaína mais alta $\left(150 \mathrm{mg} . \mathrm{L}^{-1}\right)$ levaram cerca de 3 minutos para a recuperação, enquanto os peixes expostos às concentrações mais baixas precisaram de apenas de 1 minuto.

Antunes et al. (2008), estudando a relação da dose ótima de benzocaína e o peso de carpas (Cyprinus carpio), relatam que o aumento de $1 \mathrm{~g}$ no peso dos indivíduos corresponde a um aumento de $0,158 \mathrm{mg} . \mathrm{L}^{-1}$ na quantidade anestésica. As concentrações testadas foram de 100, 140, 180 e $220 \mathrm{mg} \cdot \mathrm{L}^{-1}$, e todas se mostraram seguras e eficazes, não ocasionando nenhuma mortalidade.

Figura 2. Indução e recuperação da eugenol para juvenis de pacu.

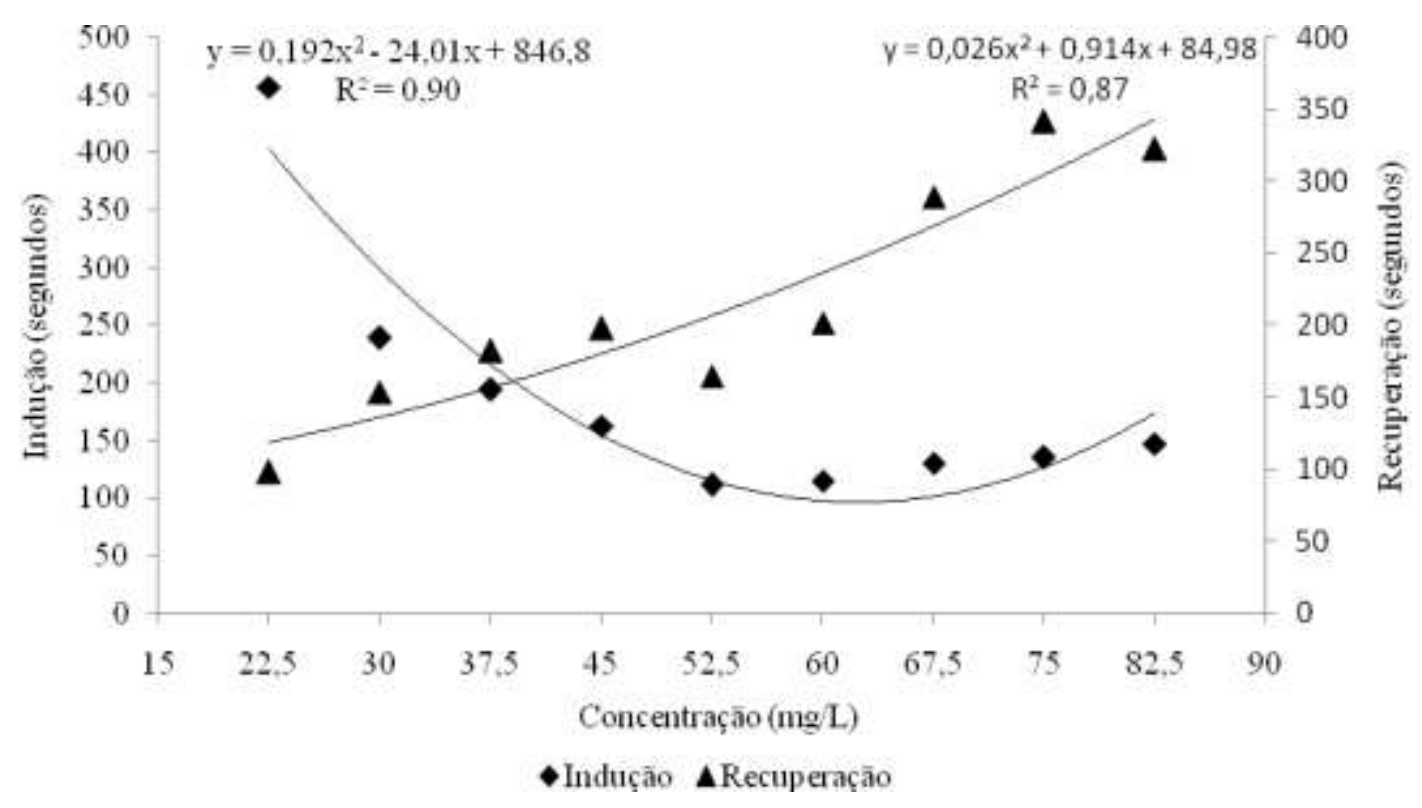

Indução: $y=0,192 x^{2}-24 \cdot 01 x+846,8, R^{2}=0,90$. Recuperação: $y=0,026 x^{2}+0,914 x+84,98, R^{2}=0,90$ Fonte: Autores.

As doses de eugenol de 7,5 e $15 \mathrm{mg} \cdot \mathrm{L}^{-1}$ não apresentaram efeito de indução. $\mathrm{O}$ efeito foi atingido somente a partir da dose de 22,5 mg. $\mathrm{L}^{-1}$. Verificou-se que ocorreu diferença estatística $(\mathrm{P}<0,05)$ entre as diferentes concentrações examinadas. Contudo, a dose de $52,5 \mathrm{mg} . \mathrm{L}^{-1}$ foi mais eficaz tanto para a indução, quanto para recuperação dos peixes (Tabela 03). Após as 96 horas de monitoramento não foi constatada mortalidade dos peixes expostos à anestesia com eugenol. 
Tabela 3. Tempo de indução e recuperação de juvenis de pacu, expostos a diferentes concentrações de eugenol.

\begin{tabular}{lcc}
\hline Concentração $\left(\mathrm{mg} . \mathrm{L}^{-1}\right)$ & Indução (segundos) & Recuperação (segundos) \\
\hline 22,5 & $456,9 \pm 87,4 \mathrm{a}$ & $98,4 \pm 14,5 \mathrm{f}$ \\
30,0 & $238,8 \pm 22,4 \mathrm{~b}$ & $153,9 \pm 16,2 \mathrm{def}$ \\
37,5 & $194,0 \pm 21,6 \mathrm{bc}$ & $182,1 \pm 20,0 \mathrm{def}$ \\
45,0 & $162,8 \pm 18,4 \mathrm{~cd}$ & $197,9 \pm 21,5 \mathrm{de}$ \\
52,5 & $111,4 \pm 19,1 \mathrm{e}$ & $164,7 \pm 23,5 \mathrm{def}$ \\
60,0 & $114,8 \pm 17,8 \mathrm{e}$ & $201,8 \pm 26,9 \mathrm{~d}$ \\
67,5 & $130,5 \pm 28,6 \mathrm{de}$ & $289,5 \pm 80,6 \mathrm{abc}$ \\
75,0 & $135,5 \pm 25,1 \mathrm{e}$ & $341,3 \pm 115,5 \mathrm{a}$ \\
82,5 & $147,2 \pm 22,3 \mathrm{cde}$ & $322,6 \pm 129,3 \mathrm{ab}$ \\
\hline C.V. $(\%)$ & 19,1 & 30,41 \\
\hline
\end{tabular}

Medias na mesma coluna seguidos de mesma letra não diferem estatisticamente $(\mathrm{P}<0,05)$ pelo teste de Tukey.

Fonte: Autores.

As respostas observadas para juvenis de pacu, concordam com os obtidos por Gonçalves et al. (2008) que avaliaram a eficiência de anestésicos naturais na indução de juvenis de pacu, os quais relatam que a melhor dose encontrada foi de 50 mg.L $\mathrm{L}^{-1}$, concentração similar a 52,5 mg.L $\mathrm{L}^{-1}$ encontrado no presente estudo. Rotili et al. (2012) avaliando diferentes dosagens de eugenol (40, 80, 120, 160 e $\left.200 \mathrm{mg} . \mathrm{L}^{-1}\right)$ para diferentes pesos de pacus (P. mesopotamicus) (50, 100, 150, 200 e 250 gramas), observaram que o maior tempo de indução para $40 \mathrm{mg} . \mathrm{L}^{-1}$, para os peixes com 50 gramas e o menor tempo para os peixes com 100 gramas na dosagem de 200mg. $\mathrm{L}^{-1}$, contudo este autores, relatam que o menor tempo de recuperação foi obtido com 80mg.L $\mathrm{L}^{-1}$, para os peixes com 50, 100 e 150 gramas, e $40 \mathrm{mg} . \mathrm{L}^{-1}$ para as classes de 200 e 250 gramas, observaram ainda que o menor tempo de recuperação não está diretamente relacionado ao menos tempo de indução.

Notou-se que o coeficiente de variação da indução anestésica foi menor $(19,1 \%)$ do que o da recuperação $(30,41 \%)$. Esse coeficiente de indução e recuperação foram semelhantes aos observados em tilápia-do-nilo (Oreochromis niloticus), 26,96 e 34,17\% (Vidal et al., 2008); matrinxã (Brycon cephalus), 13,86 e 24,84\% (Inoue et al., 2003); tambaqui (Colossoma macropomum), 24,54 e 44,67\% (Roubach et al., 2005) e piavuçu (Leporinus macrocephalus), 16,91 e 22,01\% (Vidal et al., 2007).

O tempo ideal para a recuperação dos peixes deve ser inferior a 5 minutos (Roubach \& Gomes, 2001). Contudo, as doses de 75 e 82,5 mg.L.-1 estão acima desse intervalo de tempo indicando que ficam fora da margem de segurança da concentração ideal, portanto é aconselhável empregar doses menores. Porém, os resultados divergem dos apresentados por Gonçalves et al. (2008) para a mesma espécie, os autores constataram que na concentração de $100 \mathrm{mg} . \mathrm{L}^{-1}$ de eugenol, o tempo de recuperação médio foi de três minutos. A divergência deve estar relacionada com a temperatura, visto que, Hoskonen e Pirhonen (2004) afirmam que a temperatura apresenta forte influência sobre o efeito anestésico do óleo de cravo nos peixes.

Segundo Vidal et al. (2006) a dose de $50 \mathrm{mg} . \mathrm{L}^{-1}$ é suficiente para a indução anestésica de juvenis de pintado (Pseudoplatystoma coruscans). Assim, recomenda o seu emprego no manejo desses animais uma vez que esse método facilita o manuseio e não apresenta efeitos adversos aparentes à saúde dos mesmos, dose semelhante a 52,5 mg. $\mathrm{L}^{-1}$ encontrado no atual estudo. 
Barbosa et al. (2007) verificaram que o eugenol não reduziu totalmente as reações ao estresse, quando avaliaram as respostas metabólicas do matrinxã (Brycon amazonicus) submetido a banhos anestésicos. Contudo, não provocou estresse adicional, sendo, portanto, adequado seu emprego para a espécie.

\section{Conclusão}

A concentração de benzocaína de $75 \mathrm{mg} \cdot \mathrm{L}^{-1} \mathrm{e}$ a concentração de eugenol de $52,5 \mathrm{mg} \cdot \mathrm{L}^{-1}$ são indicadas para indução e recuperação de juvenis de pacu (Piaractus mesopotamicus).

Contudo, ainda são necessários mais estudos a respeito da utilização da benzocaína e do eugenol para o pacu, pois dependendo da concentração e do tempo de exposição utilizado, o próprio anestésico pode ser prejudicial aos peixes, com consequiências desastrosas, como morte instantânea dos animais.

\section{Referências}

Antunes, M. I. P. P., Spurio, R. S., Godoi, D. A., Grumadas, C. E. S., \& Rocha, M. A. (2008). Cloridrato de benzocaína na anestesia de carpas (Cyprinius carpio). Semina Ciências Agrárias, 29(1), 151-156.

Anziliero, D., Kreutz, L. C., \& Barcellos, L. J. G. (2008). Avaliação de quatro diferentes anestésicos para sua utilização no manejo e jundiás (Rhamdia Quelen). In: 35 CONBRAVET, 2008, Gramado. Anais 35 CONBRAVET.

Barbosa, L. G., Moraes, G., \& Inoue, L. A. K. (2007). Respostas metabólicas do matrinxã submetidos a banho anestésico de eugenol. Acta Scientiarum, 29(3), $255-260$.

Bombardelli, R. A., Bencke, B. C., \& Sanches, E. A. (2007). Processamento da carne do pacu em tanques--rede no reservatório de Itaipu-rede no reservatório de Itaipu. Acta Scientiarum. 29(4), 457-463.

Coldebella, A., Godoy, A. C., Gentelini, A. L., Piana, P. A., Coldebella, P. F., Boscolo, W. R., \& Feiden, A. (2020) Nitrogen and phosphorus dynamics in Nile tilapia farming in excavated rearing ponds. Research, Society and Development, 9(1), e1319119699.

Faria, R. H. S., Souza, M. L. R., Wagner, P. M., Povh, J. A., \& Ribeiro, R. P. (2003). Rendimento do processamento da tilápia do Nilo (Oreochromis niloticus Linnaeus, 1757) e do pacu (Piaractus mesopotamicus) Holmberg, 1887). Acta Scientiarum. 25(1), 21-24.

Fernandes, J. B. K., Carneiro, D. J., \& Sakomura, N. K. (2000). Fontes e níveis de proteína bruta em dietas para alevinos de pacu (Piaractus mesopotamicus), Revista Brasileira de Zootecnia, 29(3), 646-653.

Gimbo, R, Y., Saita, M. V., Gonçalves, A. F. N., \& Takahashi, L. S. (2008). Diferentes concentrações de benzocaína na indução anestésica do lambari-do-rabo amarelo (Astyanax altiparanae). Revista Brasileira de Saúde e Produção Animal, 9(2), 350-357.

Gomes, L. C., Chippari-Gomes, A. R., Lopes, N. P., Roubach, R., \& Araujo-Lima, C. A. R. M. (2001). Efficacy of benzocaine as an anesthetic in juvenile tambaqui Colossoma macropomum. Journal of the World Aquaculture Society, 32(4), 426-431.

Gonçalves, A. F. N., Santos, E. C. C., Fernandes, J. B. K., \& Takahashi, L. S. (2008). Mentol e eugenol como substitutos da benzocaína na indução anestésica de pacu. Acta Scientiarum, 30(3), 339-344.

Hajek, G. J., Klyszejko, B., \& Dziaman, R. (2006). The anaesthetic effect of clove oil on common carp, Cyprinus carpio L. Acta Ichthyologica et Piscatoria, 36, 93-97.

Hisano, H., Ishikawa, M. M., Ferreira, R. A., Bulgarelli, A. L. A., Costa, T. R., \& Pádua, S. B. (2008). Tempo de indução e de recuperação de dourados Salminus brasiliensis (Cuvier, 1816), submetidos a diferentes concentrações de óleo de cravo Eugenia sp. Acta Scientiarum, 30(3), $303-307$.

Hoskonen, P., \& Pirhonen, J. (2004). Temperature effects on anaesthesia with clove oil in six temperate-zone fishes. Journal of Fish Biology, 64, 1136-1142.

Inoue, L. A. K. A., Santos-Neto, C., \& Moraes, G. (2003). Clove oil as anaesthetic for juveniles of matrinxã Brycon cephalus (Gunther, 1869). Ciência Rural, 33(5), 943-947.

Jomori, R. K., Carneiro, D. J. Martins, M. I. E. G., \& Portella, M. C. (2005). Economic evaluation of Piaractus mesopotamicus juvenile production in different rearing systems. Aquaculture, 243, 175-183.

Nakatani, K., Agostinho, A. A., Baumgartner, G., Bialetzki, A., Sanches, P. V., \& Makrakis, M. C. (2001). Ovos e larvas de peixes de agua doce. Maringá: EDUEM, 378p

Okamoto, M. H., Tesser, M. B., Louzada, L. R., Santos, R. A., \& Sampaio, L. A. (2009). Benzocaína e eugenol como anestésicos para juvenis de pampo (Trachinotus marginatus). Ciência Rural, 39(3), 866-870.

Pereira, A. S., Shitsuka, D. M., Parreira, F. J., \& Shitsuka, R. (2018). Metodologia da pesquisa científica. (1ª . ed.): UFSM, NTE.

Ross, L. G., \& Ross, B. (1999). Anaesthetic and sedative techniques for aquatic animals. Oxford: Blackwell Science, 159p. 
Research, Society and Development, v. 10, n. 8, e47510817573, 2021

(CC BY 4.0) | ISSN 2525-3409 | DOI: http://dx.doi.org/10.33448/rsd-v10i8.17573

Rotili, D. A., Devens, M. A., Diemer, O., Lorenz, E. K., Lazzari, R., \& Boscolo, W. R. (2012) Uso de eugenol como anestésico em pacu. Pesquisa Agropecuária Tropical, 42 (3), 288-294.

Roubach, R., \& Gomes, L. C. (2001) O uso de anestésicos durante o manejo de peixes. Panorama da aqüicultura, 11, 37-40.

Roubach, R., Gomes, L. C., Fonseca, F. A. L., \& Val, A. L. (2005). Eugenol as an effi cacious anaesthetic for tambaqui, Colossoma macropomum (Cuvier). Aquaculture Research, 36, 1056-1061.

Signor, A. A., Boscolo, W. R., Feiden, A., Bittencourt, F., Coldebella, A., \& Reidel, A. (2010) Proteína e energia na alimentação de pacus criados em tanquesrede. Revista Brasileira de Zootecnia, 39(11), 2336-2341.

Signor, A. A., Reidel, A., Coldebella, A., Ferreira, H. K. A., Signor, F. R. P., Buglione Neto, C. C., \& Watanabe, A. L. (2020). Densidade de estocagem de jundiá (Rhamdia quelen) em tanques-rede na fase inicial. Brazilian Journal of Development. v.6 (7).

Tavares, S. G., Queiroz, S. S., Bertolini, G. R. F., Grandi, A. M., Rodrigues, M. L., \& Signor, A. (2021) World scientific production on biofloc technology in tilapiculture as a driver of sustainable development. Research, Society and Development, 10(5), e56910515200.

Urbinati, E. C., \& Gonçalves, F. D. (2005). Pacu (Piaractus mesopotamicus). In: Baldisseroto, B., Gomes, L. C. (Ed.). Espécies nativas para piscicultura no Brasil. Santa Maria: UFSM, 225-246.

Vidal, L. V. O., Albinati, R. C. B., Luscher, A. C., \& Mecêdo, G. R. (2006). Utilização do eugenol como anestésico para o manejo de juvenis de Pintado (Pseudoplatystoma coruscans). Acta Scientiarum, 28(3), 275-279.

Vidal, L. V. O., Albinati, R. C. B., Albinati, A. C. L., Lira, A. D., Almeida, T. R., \& Santos, G. B. (2008). Eugenol como anestésico para a tilápia-do-nilo. Pesquisa Agropecuária Brasileira, 43(8), 1069-1074.

Vidal, L. V. O., Furuya, W. M., Graciano, T. S., Schamber, C. R., Santos, L. D., \& Soares, C. M. (2007). Concentrações de eugenol para anestesia profunda e toxidade aguda em juvenis de piavuçu (Leporinus macrocephalus). Acta Scientiarum, 29, 357-362.

Woody, C. A., Nelson, J., \& Ramstad, K. (2002). Clove oil as an anaesthetic for adult sockeye salmon: Field trials. Journal of Fish Biology, 60(2), $340-347$. 